\title{
PReF: describing key Preprint Review Features
}

Authors: Jessica Polka*1 (iD , Iratxe Puebla*1 (iD, Damian Pattinson*2 (iD, Phil Hurst ${ }^{* 3}$ iD, Gary S. McDowell ${ }^{\star 4,5}$ (iD), Richard Sever*6 (iD , Thomas Lemberger ${ }^{7}$ (D), Michele Avissar-Whiting $^{8}$ (iD , Philip N. Cohen ${ }^{9}$ iD , Tony Ross-Hellauer ${ }^{10}$ (iD), Gabe Stein ${ }^{5}$ (iD), Kathleen Shearer ${ }^{11}$ (D) , Clare Stone ${ }^{12}$ iD , Victoria T. Yan ${ }^{1,13}$ iD

*These authors contributed equally to this work.

Correspondence: Victoria T. Yan (victoria.yan@embl.de)

Author affiliations:
1. ASAPbio
6. Cold Spring Harbor
10. TU Graz
2. elife Laboratory
11. COAR
3. Royal Society
7. EMBO
12. SSRN
4. Lightoller
8. Research Square
13. Presently at EMBL
5. Doc Maps
9. SocArxiv

\begin{abstract}
Preprints catalyze rapid and open communication of research. A frequent criticism of preprints, however, is their lack of peer review. In recent years, myriad new initiatives have enabled review of preprinted research to be coordinated, collected, and displayed alongside preprints. This provides evaluation and context for readers, as well as feedback for the authors. The processes behind preprint review are diverse and may differ from journal peer review, which can be a challenge for readers seeking to compare and interpret the reviews. To address this, the ASAPbio organized a working group that set out to define key features of preprint review processes. Here, we describe Preprint Review Features (PReF) as descriptors and provide an implementation guide. PReF captures the key elements of preprint review processes using 8 standard key-value pairs. PReF can serve within the descriptions of individual preprint review processes, and act as search filters on indexing services. Widespread adoption of PReF will promote understanding and categorization of preprint review and improve its discoverability.
\end{abstract}

\section{Introduction}

Preprints enable innovations in transparent and open peer review and new forms of early feedback and evaluation (1). These new initiatives span a wide range of review activities - formal or informal - driven by the community or coordinated by journals. These projects are heterogeneous and constantly evolving and sometimes very different from journal peer review processes that researchers are familiar with. This presents a challenge for authors, reviewers, journal editors, and readers, who need to know how these new forms of reviews relate to, and 
differ from, traditional review models. The lack of consistent language and a framework to help define these initiatives makes it difficult to understand how new projects fit into the ecosystem and gauge how preprint review activities are evolving in terms of their adoption and usage. In addition, we acknowledge the inherent ambiguity in the terms "feedback," "commenting" and "peer review;" therefore, we aimed to describe and understand the basic elements that characterize a broad range of review processes for preprints.

Notably, journal peer review processes also vary and are not standardized across journals and disciplines $(\underline{2}, \underline{3})$. Prior efforts to describe peer review have done so in the context of traditional publishing. The Peer Review Transparency project (4) proposed a peer review taxonomy with a broad scope that includes books, the STM Peer Review terminology describes journal peer review (므), and the Open Peer Review taxonomy describes the major attributes of open peer review (1). Here, we aimed to address the new axes that emerge when we move to the preprint space. Preprints enable peer review to take place without coordination by a journal and be organized instead by the research community independently of the journal publishing process. Preprints also enable the contribution of reviews independently of the authors' permission or an editor's invitation. There is a need to ensure clear expectations and practices around the process of peer review in a world where publishing and review are increasingly separated from curation (ㅁ).

The emergence of a multitude of platforms for posting review on preprints creates the need to aggregate review. Preprint review aggregators like Early Evidence Base and Sciety and server-based aggregation like the bioRxiv dashboard can assemble and alert readers to content from different preprint review platforms and communities. Both systems use the machine-readable DocMaps framework to record and exchange information about preprint reviews ( $\underline{7})$, but this is not designed to characterize differences among preprint review processes that would allow review activities to be consistently interpreted, compared, and tracked.

In order to characterize the differences between preprint review processes, ASAPbio convened a working group to develop a description of features to help readers interpret the processes behind preprint review. The group involves representatives from preprint servers, preprint review platforms and aggregators, technologists, and publishers (see member details in working group section below). The goal was to develop Preprint Review Features (PReF) that describe the key elements of preprint review activities for human readers in a way that is simple for aggregators, platforms, and others to implement. We focused mainly on the life sciences and excluded initiatives designed primarily for curation rather than evaluation (e.g., Peeriodicals). We envision PReF being used to describe the processes behind the review, rather than individual reviews, comments, or evaluations themselves.

We aimed to develop consistent descriptions that would provide a more granular understanding of the features of the preprint review process than is provided by more ambiguous terms such as 'commenting,' 'community review,' 'post-publication review,' or 'overlay journal.' By displaying the PReF fields, a platform can reveal elements of the review activity that has taken place for 
preprints it hosts. These features could also function as a search filter for indexing services to enhance discoverability of preprints with certain types of review. Furthermore, structured information about preprint review projects will enable the comparison of services with one another and the tracking of broader review activity trends. Ultimately, clarity about the types and extent of evaluation that preprints undergo should build trust in preprints and the evolving review ecosystem.

PReF covers preprint review activities wherever they take place, regardless of whether the main goal of the platform hosting the review is to coordinate such activities. The description is structured as a set of standardized key-value pairs: for each field, a finite number of pre-determined values are defined. Each field is described as a question and its possible values are the respective formalized answers to this question. For the sake of simplicity, we use terms such as "Review" and "Reviewer"; however, we view PReF as encompassing a spectrum of feedback interactions and evaluations of preprint articles. While not all of the processes described by the features may be universally considered to be "reviews," we aim to surface and clarify highly structured and moderated review processes as distinct from more informal feedback.

\section{PReF Fields}

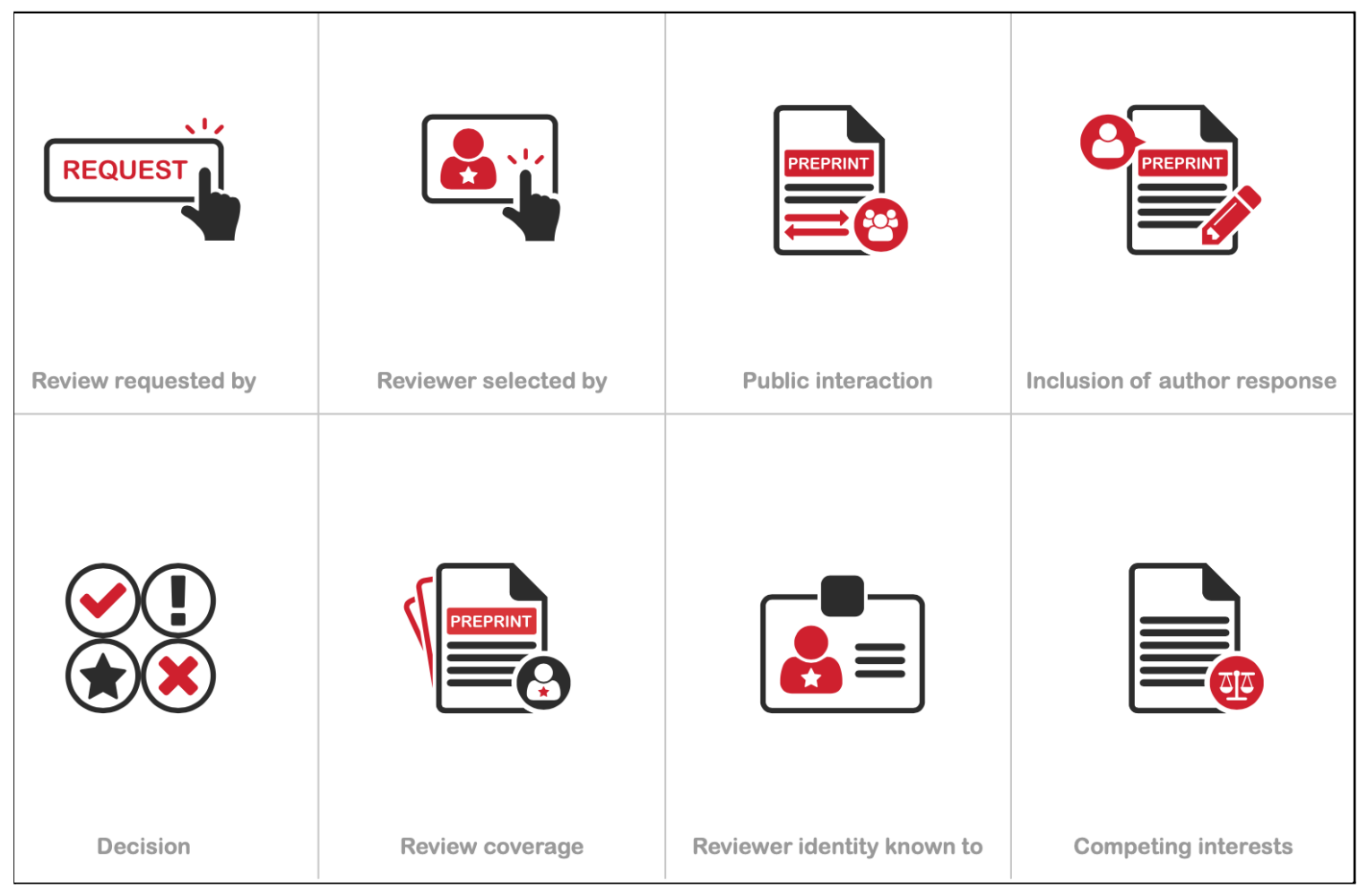

Figure 1. Icons for PReF fields. We encourage the use of these icons alongside PReF. 
Multiple entry options are possible for each field, for example, in cases where multiple workflows are available.

\begin{tabular}{|l|l|l|}
\hline Name of Field & Description & Options for entry \\
\hline Review requested by & Who initiates the review process? & $\begin{array}{l}\text { Authors } \\
\text { Non-authors }\end{array}$ \\
\hline Reviewer selected by & Who selects the review providers? & $\begin{array}{l}\text { Authors } \\
\text { Editor, service, or } \\
\text { community } \\
\text { Self-nominated }\end{array}$ \\
\hline Public interaction & $\begin{array}{l}\text { Is there an opportunity for the public to } \\
\text { engage as an integral part of the } \\
\text { process? }\end{array}$ & $\begin{array}{l}\text { Yes } \\
\text { No }\end{array}$ \\
\hline $\begin{array}{l}\text { Inclusion of author } \\
\text { response }\end{array}$ & $\begin{array}{l}\text { Is the author's response included as an } \\
\text { integral part of the process? }\end{array}$ & $\begin{array}{l}\text { Yes } \\
\text { No }\end{array}$ \\
\hline Decision & $\begin{array}{l}\text { Does the service provide a binary } \\
\text { decision (accept/reject or } \\
\text { recommendation) or a rating after the } \\
\text { review process? }\end{array}$ & $\begin{array}{l}\text { Binary decision } \\
\text { Other scale or rating } \\
\text { None }\end{array}$ \\
\hline $\begin{array}{l}\text { Review coverage } \\
\text { to }\end{array}$ & $\begin{array}{l}\text { Does the review cover the entire paper or } \\
\text { only a certain section or aspect? }\end{array}$ & $\begin{array}{l}\text { Complete paper } \\
\text { Specific aspects }\end{array}$ \\
\hline $\begin{array}{l}\text { Are the identities of reviewers known to } \\
\text { everyone (public), editors or service, or } \\
\text { no one? }\end{array}$ & $\begin{array}{l}\text { Is a competing interest statement } \\
\text { provided? } \\
\text { Editor or service } \\
\text { No one }\end{array}$ \\
\hline
\end{tabular}

\section{Detailed definitions for PReF fields}

\section{Review requested by}

Who initiates the review process?

This field designates the party that initiates the request for review of the preprint. Unlike traditional journals, preprint review can take place independently of the authors of the preprint. There is no requirement for the authors to submit a request for review in order for an individual or group to choose to review it. In situations where the review is initiated by the authors, this may require the submission of the manuscript to the preprint review service or platform. 
- Authors - The authors of the paper submit it for review by the service. Examples include journals that coordinate preprint review (e.g. eLife) and platforms such as Review Commons and Peer Community In.

- Non-authors - The review process is initiated by someone other than the authors of the paper, such as an individual reader or the curators or editors of reviewing services.

Examples include a reader commenting on a preprint server, Rapid Reviews: COVID-19, and preLights.

\section{Reviewer selected by}

Who selects the reviewers?

This field designates the party who coordinates and/or has oversight over the selection of the individuals who review the preprint.

- Authors - Reviewers are selected or nominated by the authors of the paper. Examples include F1000 Research and Wellcome Open Research.

- Editor, service, or community - Reviewers are selected by the journal, service, or community overseeing the review process, not including the authors. Examples include Review Commons, Peer Community In, and eLife.

- Self-nominated - Reviewers are not invited by the authors or another party, but instead decide to participate themselves. Examples include a reader commenting on a preprint server, ASAPbio crowd preprint review, and preLights.

\section{Public interaction}

Is there an opportunity for the public to engage as an integrated part of the process?

This field designates whether the review process allows others to respond or react to review of the preprint via the same channels as those by which the preprint review was provided.

- Included - Even if there are restrictions on who can participate, members of the public can comment on the review (for example, preLights). All services that allow self-nominated participation in review also fall into this category (e.g., PREreview).

- Not included - Comments from the public are not permitted through the same channel as those used by the platform, service, or community to coordinate and/or host reviews (for example, Review Commons has its own Hypothes.is group, in which reviews are displayed, but anyone wishing to respond to those reviews would need to do so via the comment section of the preprint itself).

\section{Inclusion of author response}

Is the author's response included as an integral part of the process? 
This field designates whether the authors of the preprint can provide a response to the review of their paper via the same channels as those through which the preprint review has been provided. In some cases, the authors may be actively invited to provide a response to the review.

- Included - The review process provides an opportunity for the authors of the paper to respond, and these responses are displayed alongside the review. Examples include Review Commons, PeerRef, and eLife.

- Not included - The review process does not make accommodations for responses from authors of the paper. Authors may be able to respond by posting their own comment, but it is not an integral part of the process. Examples include Rapid Reviews: COVID-19.

\section{Decision}

Does the review process provide a binary decision (accept/reject or recommendation) or a rating after the review process?

This field describes whether the preprint review process produces a judgment or decision as an outcome. The decision outcome may be posted publicly, along with the evaluation.

- Binary decision - The service offers an accept/reject decision or a recommendation at the end of the process. Examples include Peer Community In, Plaudit, and Episciences.

- Other scale or rating - The service provides a non-binary decision, such as scale or rating. Examples include Rapid Reviews: COVID-19 and the rapid review feature of PREreview.

- None - The service does not provide a decision or rating at the end of the process. Examples include Review Commons.

\section{Review coverage}

Does the review cover the entire paper or only a certain section or aspect?

This field designates whether a review of the preprint is expected to cover the full contents of the paper or can just relate to a specific portion or aspect of it.

- Complete paper - Reviewers are expected to read and comment on the entire paper. Examples include Review Commons, Peer Community In, and eLife.

- Specific aspects - Reviewers have the option of discussing only a specific portion (such as a figure) or aspect (such as statistical rigor). Examples include ASAPbio crowd preprint review and Research Square Reporting Badges.

\section{Reviewer identity known to}

Are the identities of reviewers known to everyone (public), the editors/service, or no one? 
This field designates the extent to which the identities of those providing a review of the preprint are masked or visible to the authors, each other, the journal, the service or community overseeing the review process, or the public.

- Public - The review provider has signed their name or provided other identifying information that is visible to the public. Examples include Peer Community In and Rapid Reviews: COVID-19.

- Editor or service - Individuals associated with the service, such as an editor or a service administrator, know the identity of the reviewers, even though the reviewer names are not posted publicly. Examples include PREreview and Review Commons (when reviewers do not sign their names).

- No one - Reviewers are totally anonymous, even to administrators of the service. Examples include PubPeer.

\section{Competing interests}

Is a declaration of competing interests required?

This field designates whether the platform or service requires that those providing reviews declare competing interests in relation to their involvement in the review and/or their comments, either as a statement included with their review or privately to the platform or service administrators. The field also indicates whether the platform, service, or community has processes in place, at any stage of the review process, to check the competing interests information provided, and/or display this information publicly with the preprint review. Defining what constitutes a competing interest is beyond the scope of PReF and is up to the individual platform, service, or community.

- Displayed - Reviewers are asked to declare competing interests, and their response is publicly displayed along with their review. Examples include Peer Community In.

- Checked - One of the parties associated with the platform, service, or community (e.g., an editor) reviews information about competing interests provided by the reviewer and takes appropriate action, such as excluding a reviewer with a conflict from the process. Examples include Review Commons and eLife.

- Not included - The platform, service or community does not ask reviewers to provide information about competing interests. Examples include PubPeer.

\section{Beyond the scope of PReF}

The working group discussed several potential additional items that we opted not to include in our recommendations at the present time.

One such item relates to services that focus on curation of preprints for particular communities or audiences. We decided against including curation as a field in the terms since curation does not necessarily equate to review of the content. The lines between review and curation can blur, however, and this is an area that should be revisited as the landscape evolves. 
We also discussed whether reviews contributed by humans and machines should be distinguished. Various automated tools can now check different aspects of manuscript content (e.g., text overlap or data reporting). A prominent example is the Automated Screening Working Group, which screens COVID-19 preprints with a variety of automated tools and posts reports via Hypothes.is. It is likely that we will see further development of automated tools applicable to preprints, and the need to characterize this type of activity may thus become stronger in the future.

\section{Implementation}

The PReF fields could be used in multiple ways to help readers, evaluators, authors, and reviewers understand how the reviews were produced.

\section{Preprint review registry and aggregators}

To date, the PReF fields have been implemented to describe individual review projects on three platforms: ReimagineReview (a registry of innovative peer review projects maintained by ASAPbio), Early Evidence Base (an experimental platform operated by EMBO Press and SourceData that highlights preprints with associated reviews), and Sciety (a platform that aggregates open evaluation and curation of preprints, operated by eLife).

\section{Preprint review platforms and services}

PReF offers fields that could be used consistently across preprint review processes. The fields can be adopted by review platforms and journals to explain the preprint review workflow in their review guidelines. This will benefit readers, authors, and reviewers by allowing a comparison between the characteristics of the different preprint review processes.

For those thinking of starting a new preprint review project, the fields could act as a checklist of elements to consider when designing their preprint evaluation workflow.

\section{Readers, authors, reviewers, and other stakeholders}

For individuals who are reading preprint reviews, authors considering submitting their preprint for review, or reviewers looking for a service to work with, the fields could help them identify services that fit their needs. For authors who consider requesting peer review of their preprint, it is important to know whether the platform provides them with the opportunity to respond to the reviews before they are posted publicly. For readers, knowing whether there was some selection of reviewers as part of the process - for example to balance expertise of the reviewers - will provide useful contextual information when interpreting the critiques raised in public reviews. 

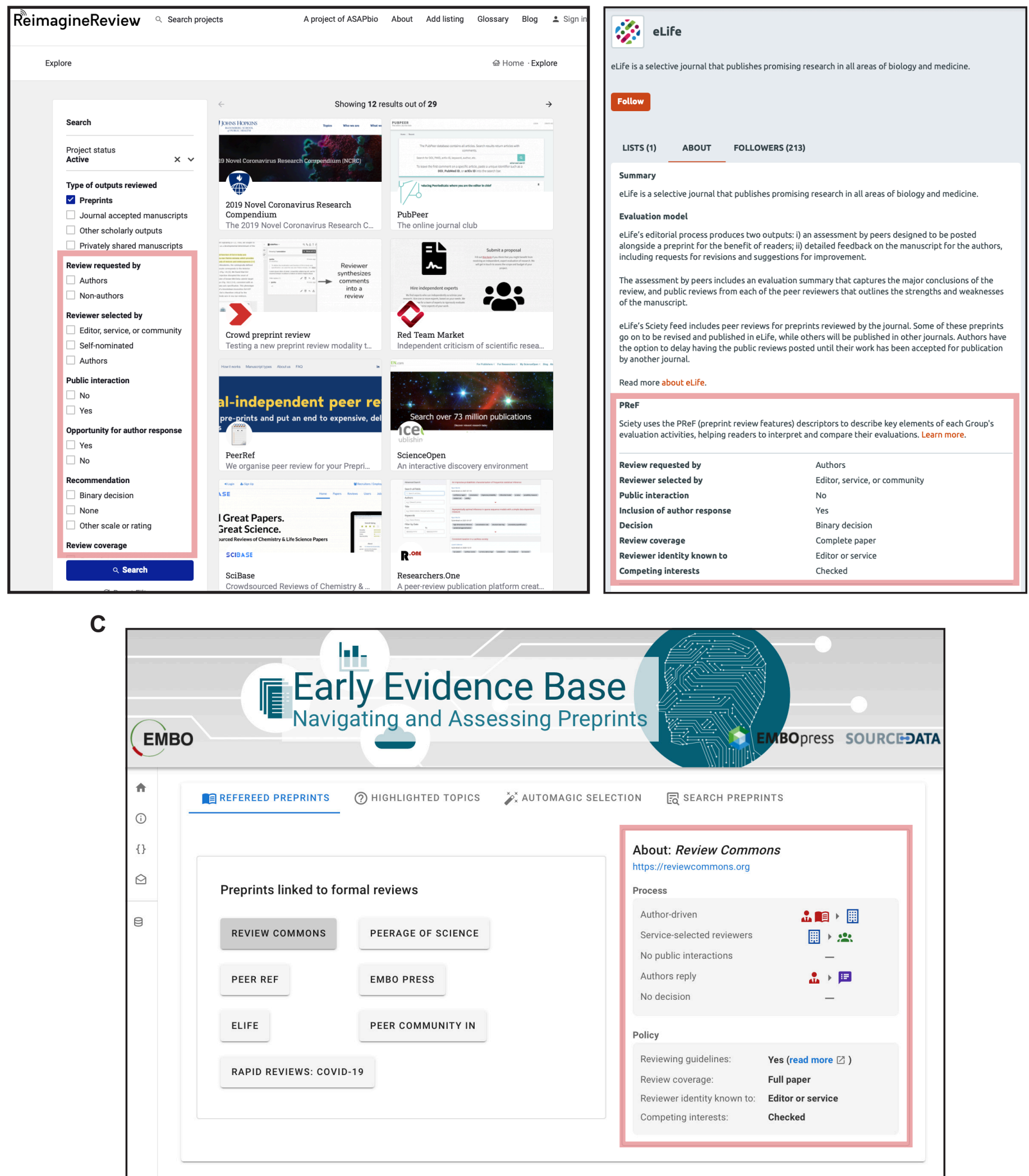

Figure 2. A. PReF implementation on ReimagineReview (https://reimaginereview.asapbio.org) demonstrating search by specific fields and combinations. B. PReF implementation on Sciety (https://sciety.org). C. PReF implementation on Early Evidence Base (https://eeb.embo.org). Red boxes highlight where PReF is used on all three projects. 
Instructors, institutions, funders, and other groups that provide recognition for or otherwise encourage peer review activity may wish to use PReF to identify suitable services. For example, an instructor of a journal-club-based class may wish to find services that enable non-authors to initiate preprint review. By making it easier to identify projects that fit various needs, PReF could facilitate recognition and credit for preprint review and commenting. Further, PReF can help institutions and funders understand the process behind the reviews on individual preprints, which can help guide their assessment of preprints.

\section{Envisioning the future}

The COVID-19 pandemic has highlighted the growing importance of preprints in the life sciences. Preprints offer unprecedented speed of dissemination, but the question for readers is how much, if at all, they have been scrutinized. By classifying a diverse range of review processes, we hope to help readers gain a better understanding of what evaluations a preprint may have received. This provides an opportunity to educate readers about what's involved in the critical assessment of scholarly work and thus build trust in preprints.

\section{Recognizing preprint review}

At present there are limited incentives for researchers to conduct review of preprints. PReF provides a step toward recognizing preprint review activity (e.g., within a researcher profile). By signaling various features of the preprint review process, the fields provide a foundation for criteria defining when a specific activity meets the requirements for inclusion in the profile as a review contribution.

A logical next step would be to incorporate PReF into a machine readable metadata standard. Making the PReF fields machine readable would be a step towards facilitating automation and the sharing of information between review platforms and other entities (e.g., ORCID), which would in turn facilitate visibility and recognition of review activities.

Another potential area of development is to enhance human readability using a graphical representation of PReF. A set of visual symbols or icons (Fig. 1B) could facilitate the integration of PReF in web pages and would provide readers with a compact depiction of the key features covered by the fields.

\section{Tracking evolution in preprint review}

The PReF terms provide a framework to understand patterns and trends within the preprint review landscape. To visualize them, we examined how current preprint review projects fall into the fields within the PReF terms (Figure 3). We examined active projects listed in

ReimagineReview as of December 1, 2021 that have a scope including review of preprints (28 projects). Social media platforms and preprint server comment sections that do not coordinate preprint review are not included. The descriptions for each project, along with an indicator of whether or not it has been verified by project leads, are archived in Zenodo and available for 
reuse. (cite: $\underline{8}$ ). This dataset can be used to compare characteristics across a wide range of platforms and services.

\section{Active Preprint Review Projects}

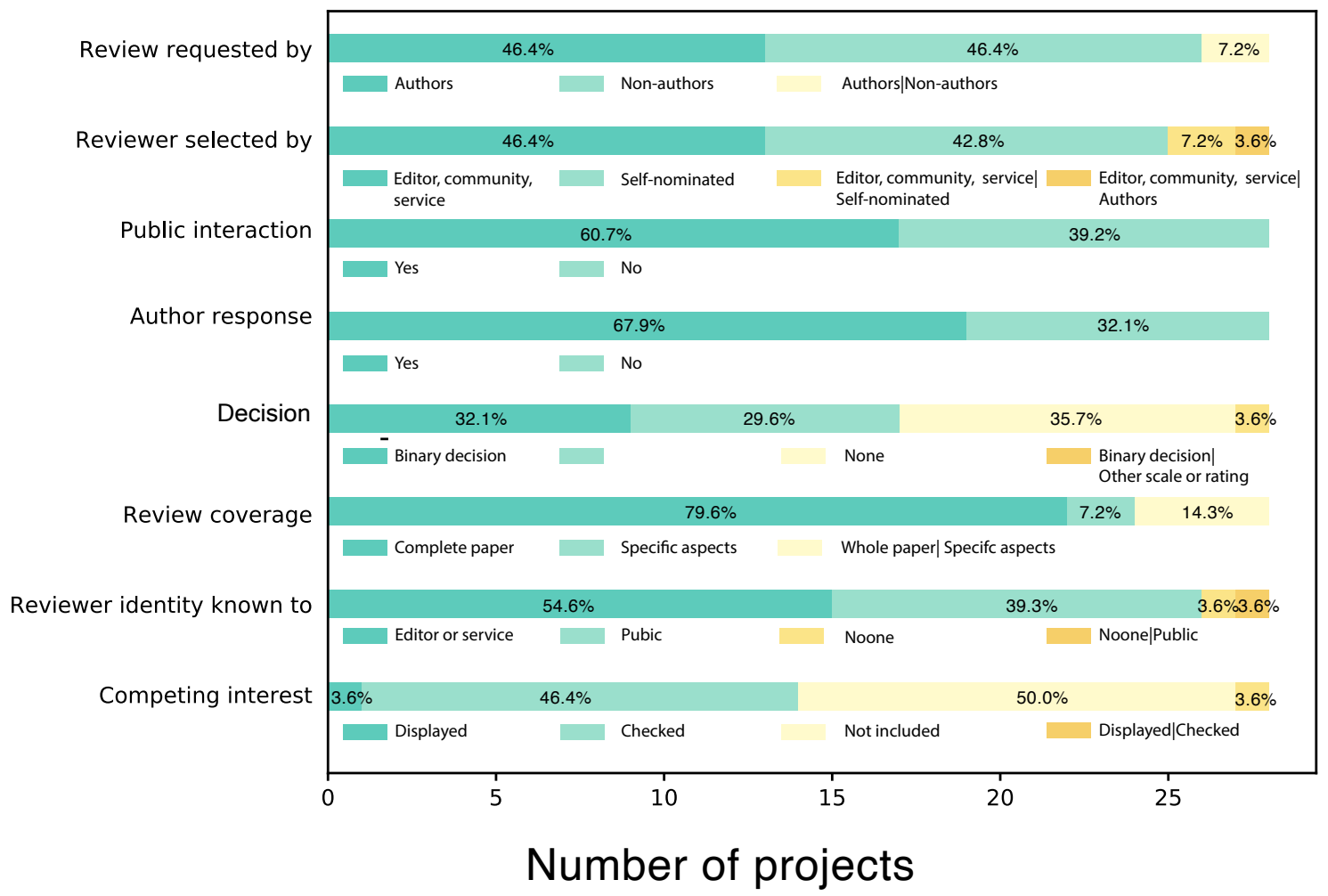

Figure 3. Active preprint review projects in RelmagineReview and their distribution in each of the PReF fields.

PReF allows abroad categorization of existing activities, which highlights a similar split between processes where reviews are driven by "Authors" and by "Non-authors" and a predominance of third-party selection of reviewer where authors initiate the review, and of self-nominated reviewers in non-author-requested review (Appendix: Figure 4). Future analysis could identify additional trends and unexplored combinations of service characteristics.

\section{How do the PReF fields relate to journal review?}

Integrating preprint review into journal processes may reduce the burden on the peer review system. At present, the diversity of preprint review models presents a challenge for their reuse, since some services deliver feedback in a format that is not equivalent to journal peer review (9). PReF helps to highlight these differences and whether the review process involves some of the elements expected as part of journal review. This can improve trust and, in situations where editors view the preprint review process as compatible with their expectations for review, facilitate the reuse of preprint review by the journal, complementing or reducing the need for additional journal peer review. 
The PReF fields were developed with preprint review in mind, but they cover a broad spectrum of review activities and are also applicable to peer review conducted by journals. In the context of journal review, PReF fields would presumably default to entries reflecting the fact that the author requests the review (in the form of submission to the journal), the selection of reviewers lies with the editor, and the review process results in a decision to accept or reject the paper. The PReF fields also touch on elements covered in earlier peer review taxonomies. For example, the STM Peer Review terminology includes reviewer transparency and post-publication commenting ( $\underline{5})$, which are analogous to the reviewer identity and public interaction elements of PReF.

Compared with earlier categorizations, PReF expands the information provided about the review process in two areas that are also applicable to journal peer review. We include a dedicated element to denote whether and how competing interests are considered during the process. While many journals have documented policies and processes for competing interests, this aspect has not featured in earlier efforts to categorize journal review. By including this element, we wish to signal the importance of considering potential competing interests and biases as part of the process. Inclusion of competing interests informs readers when these items are considered and represents an opportunity to encourage newcomers to reflect on whether declaration of competing interests is an element they should incorporate. A second new element applicable to journal review is review coverage. While peer reviews for journals have traditionally covered the full manuscript, we are seeing some specialization of reviews (e.g., statistical analyses or data review (10)) as well as new review models that incorporate greater flexibility for reviewers to comment on only specific parts of a paper (e.g., select crowd review at Synlett journal (11)). We are likely to see further experimentation around specialization and/or fragmentation within journal peer review, and PReF provides an opportunity to register this.

An important aspect of PReF is that it includes information about different actors who may be involved in the process; beyond the journal, the role of an editor or coordinator may vary or be absent, authors may or may not respond, and interactions with members of the public can and do take place.

The recent development of journals that will only review papers available as preprints (e.g., eLife, Educação em Revista) as well as initiatives to integrate preprint reviews into journals (Peer Community In (12), Review Commons) denote that we are likely to see the lines between review activities at preprints and journals blur. It is important for the PReF fields also to be applicable to journals, and we welcome feedback from editors and publishers on the elements above and on how they see PReF being applied to journals.

\section{Conclusion}

A major challenge for the widespread adoption of preprint review is a lack of understanding of the diverse processes that generate such reviews. PReF describes a set of features that allow us to distinguish, characterize, and relate preprint review processes. Alongside the fields, we provide example implementations on ReimagineReview and EEB and discuss opportunities for implementation by various stakeholders in preprint review. 
We recommend the adoption of PReF where preprint review is displayed or created. A better understanding of the variety of preprint review processes can improve trust in preprints and holds the potential to enhance recognition and re-usability of preprint reviews. Altogether, we hope that PReF can be a step toward realizing the full potential of transparent preprint review. 


\section{Appendix:}

\section{Comparison of Author, Non-author and mixed approach review processes}

Preprint review services that organize review independently of an author request represent a form of peer review enabled by preprints. Currently, the same number of projects host reviews driven by "Authors" and by "Non-authors" - 46.4\% (13 projects) in both cases - and 7.2\% (2 projects) adopt a combined approach.

In projects where authors initiate the review process, we note that reviewers are predominantly selected by a designated third party (editor, community, or service 69\%, 9/13 services), whereas the reviewers are predominantly self-nominated in non-author-requested review $(77 \%, 10 / 13$ services). In our sample, almost all non-author requested review services include public interaction as an integral part of the review process $(92 \%, 12 / 13$ services) compared with a minority for services where authors request review (23\%, 3/12 services). For the opportunity for author response, we observe the opposite trend. All author-requested review services provide an opportunity for authors to respond as an integral part of the review process $(100 \%, 13 / 13$ services), compared with $38 \%$ of non-author-requested review services (5/8). Furthermore, $85 \%$ of author-requested services check or display competing interests, whereas only $30 \%$ of non-author requested services check for competing interests.

Other factors did not vary when comparing the groups based on who initiates the review process. Approximately half of all processes initiated by authors (7/13) as well as those that are not (6/13) provide a decision as an outcome of the process. Furthermore, for both author and non-author-requested review, most services cover the full paper (12/13 in author-requested services, 9/13 only full paper, and 2/13 full paper option in the case of non-author requested services). Long-term tracking using the PReF terms may pave the way for meta-research to study preprint review, its prevalence, characteristics, and emerging trends in the ecosystem. 
A

Who selects reviewers in author vs. non-author requested and mixed approach projects?

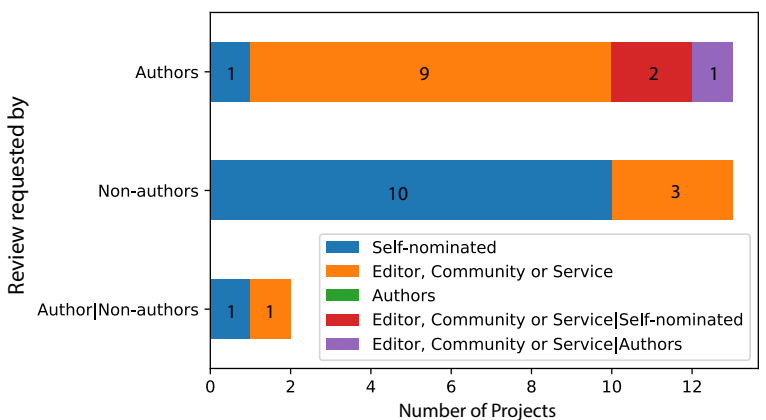

C

Inclusion of author response in author vs. non-author requested and mixed approach projects

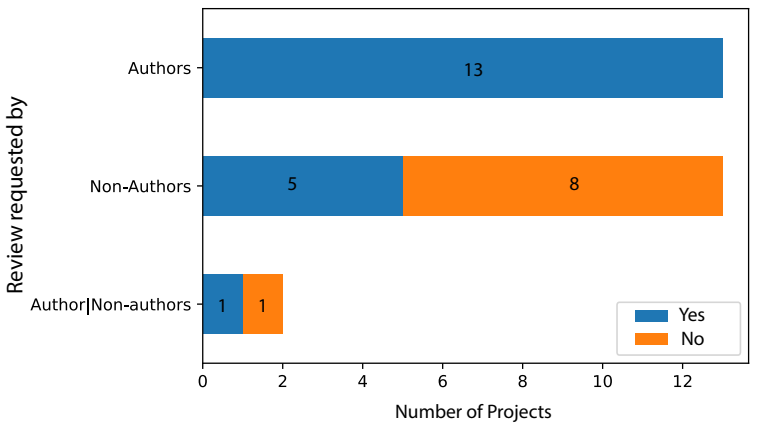

$\mathbf{E}$

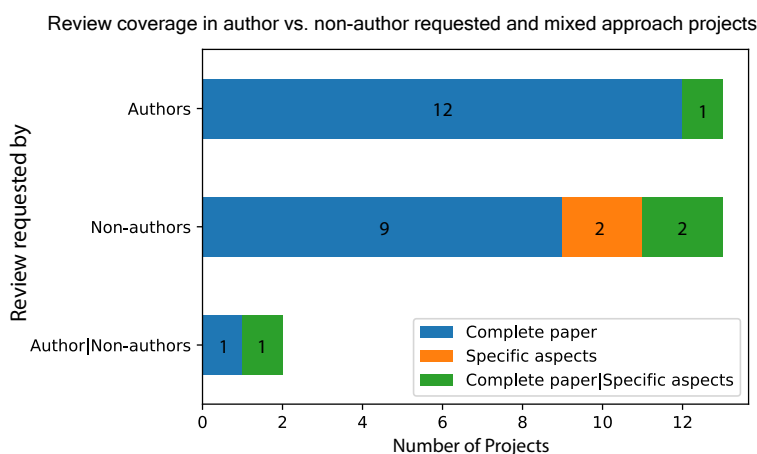

G

Inclusion of competing interest in author vs. non-author requested and mixed approach projects?

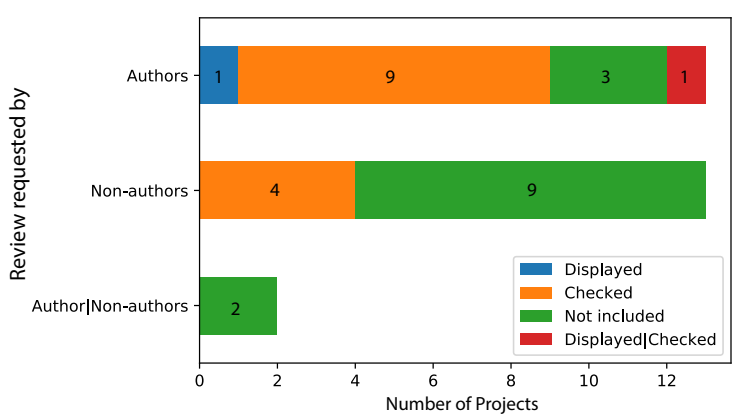

B

Inclusion of public interation in author vs. non-author requested and mixed approach projects

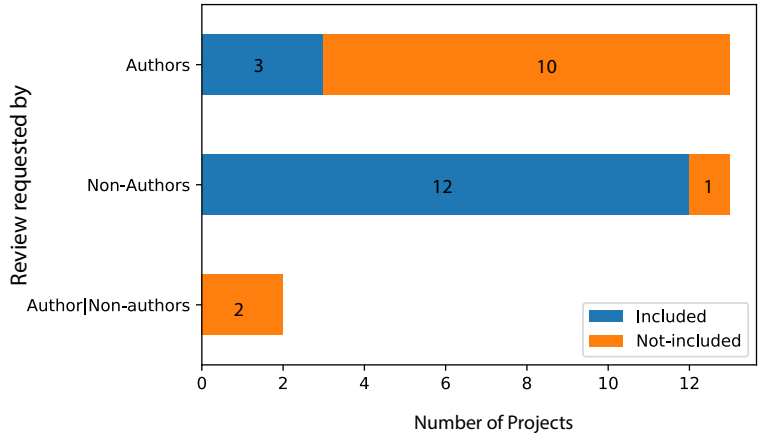

D

Inclusion of competing interest in author vs. non-author requested and mixed approach projects?

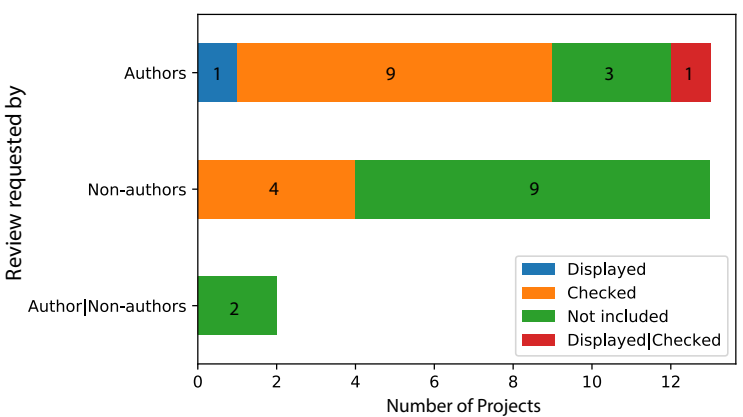

$\mathbf{F}$

Who is reviewer identity known to in author vs. non-author requested and mixed approach projects?

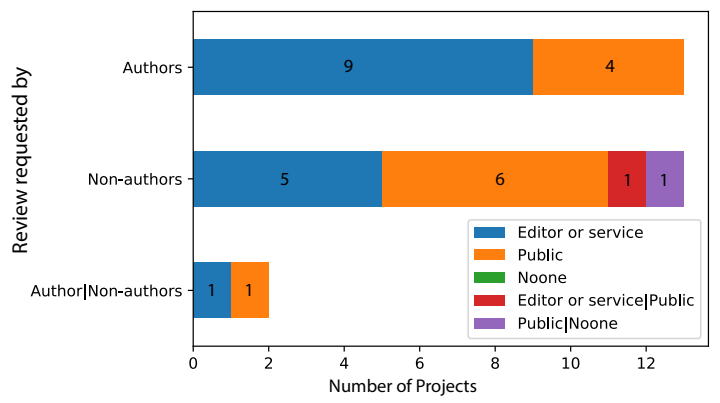

Figure 4. A. Who selects reviewers in author-requested preprint review projects compared with non-authors, and mixed author|non-author-requested review? B. Is public interaction included in author-requested preprint review projects compared with non-authors, and mixed author|non-author-requested review? C. Is the opportunity for author response included in author-requested preprint review projects compared with non-authors, and mixed author|non-author-requested review? D. Inclusion of a review decision in authorrequested preprint review projects compared with non-authors, and mixed author|non-author-requested review. E. The distribution in the types of review coverage in author-requested preprint review projects compared with non-authors, and mixed author|non-author-requested review. F. Who is reviewer identity known to, in author-requested preprint review projects compared with non-authors, and mixed author|nonauthor-requested review? G. Is competing interest displayed, checked, or not included in author-requested preprint review projects compared with non-authors, and mixed author|non-author-requested review? 


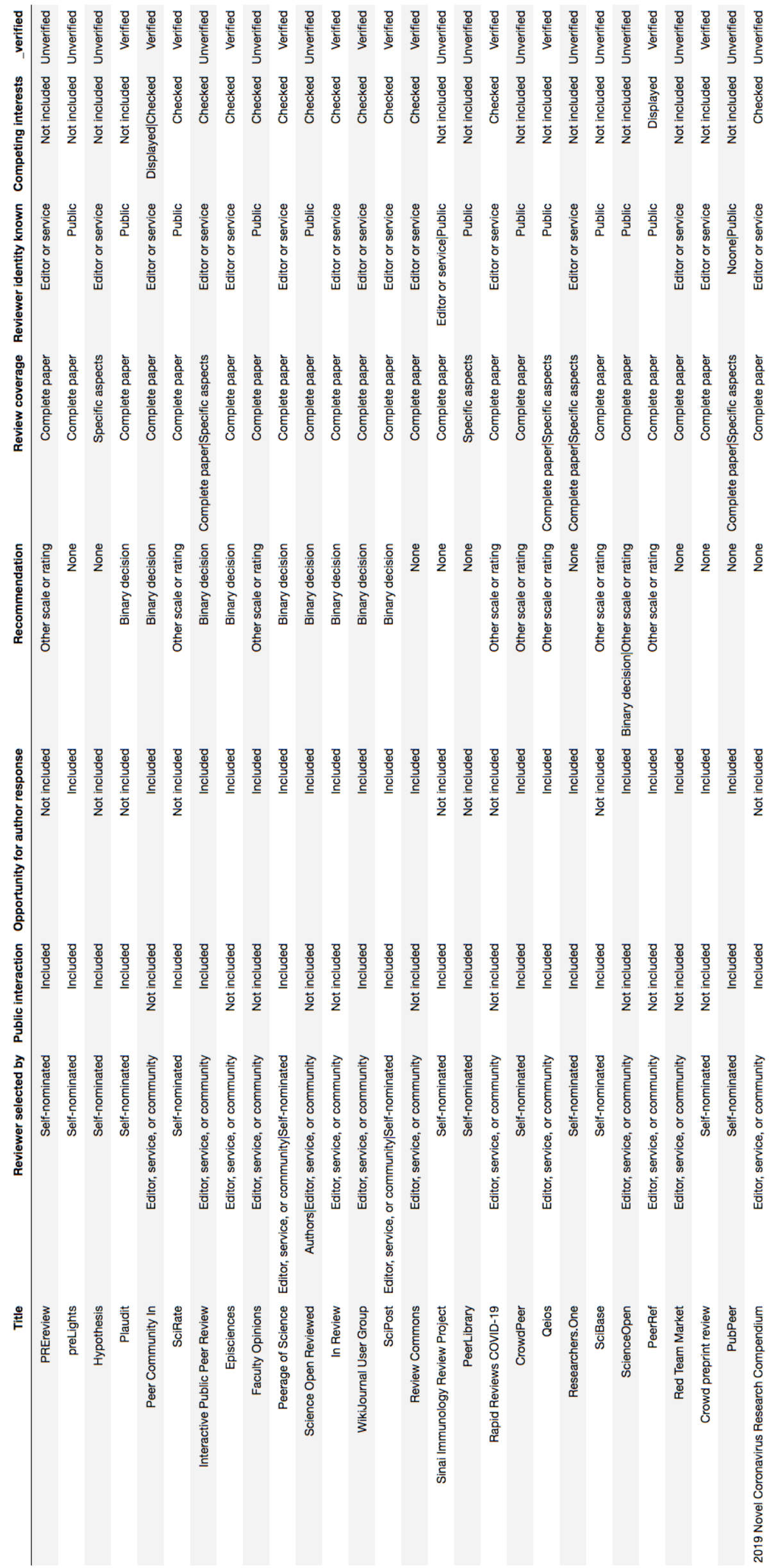

Table 1: List of preprint review services.

Link to ReimagineReview quarterly exported data on zenodo (https://zenodo.org/record/5618257). Link to analysis code and data used for the appendix (https://github.com/victoriatjyan/ReimagineReview). 


\title{
Working group members (listed alphabetically)
}

\author{
Michele Avissar-Whiting (Research Square) \\ Philip N. Cohen (SocArxiv) \\ Tony Ross-Hellauer (TU Graz/Doc Maps) \\ Phil Hurst (Royal Society) \\ Thomas Lemberger (EMBO/EEB/Review Commons) \\ Gary McDowell (Lightoller/Doc Maps) \\ Damian Pattinson (eLife) \\ Jessica Polka (ASAPbio) \\ Iratxe Puebla (ASAPbio) \\ Richard Sever (Cold Spring Harbor Laboratory and bioRxiv/medRxiv) \\ Kathleen Shearer (COAR Notify) \\ Gabe Stein (KFG/Doc Maps) \\ Clare Stone (SSRN) \\ Victoria T. Yan (ASAPbio, presently EMBL)
}

\section{Competing interests:}

Jessica Polka, Iratxe Puebla and Victoria Yan work for ASAPbio, a non-profit organisation dedicated to promoting transparency and innovations in scientific communication, including the use of preprints and preprint review. Richard Sever is a Co-Founder of the non-profit preprint servers bioRxiv and medRxiv. Michele Avissar-Whiting is editor in chief of Research Square. Damian Pattinson is Executive Director of eLife. Gary McDowell is a consultant at Lightoller LLC providing consulting services on preprint peer review. Thomas Lemberger is Deputy Head of Scientific Publications at EMBO and leads the development of the preprint peer review platform Review Commons. Clare Stone works for SSRN, a preprint server owned by Elsevier. Other authors declare no competing interests.

All these individuals acknowledge that their participation and contribution does not commit their organization to endorse these recommendations.

\section{Author contributions:}

J.P., D.P., R.S., V.Y., conceptualized the project. J.P., I.P., and V.Y., supervised the project. J.P., I.P., D.P., P.H.,G.M., and V.Y. wrote the original draft. All authors contributed to the methodology, reviewing and editing of the draft. V.Y. performed data collection, formal analysis and project administration.

\section{Acknowledgements:}

We acknowledge Elliott Lumb (PeerRef) and Andrea Attardi for feedback. 


\section{References:}

1. Ross-Hellauer T. (2017). What is open peer review? A systematic review [version 2; peer review: 4 approved]. F1000Research, 6:588 https://doi.org/10.12688/f1000research.11369.2

2. Tennant, J.P., Ross-Hellauer, T. (2020). The limitations to our understanding of peer review. Res Integr Peer Rev., 5:6 https://doi.org/10.1186/s41073-020-00092-1

3. Types of Peer Review. Wiley. Cited [2021 Dec 10]. Available from: https://authorservices.wiley.com/Reviewers/journal-reviewers/what-is-peer-review/types -of-peer-review.html

4. Peer Review Transparency project. Cited [2021 Dec 10]. Available from: https://www.prtstandards.org/

5. Jones, L., van Rossum, J., Mehmani, B., Black, C., Kowalczuk, M., Alam, S., ... Larkin, A. (2021). A Standard Taxonomy for Peer Review . Retrieved from osf.io/68rnz

6. Stern BM, O'Shea EK. (2019). A proposal for the future of scientific publishing in the life sciences. PLoS Biol 17,2: e3000116. https://doi.org/10.1371/journal.pbio.3000116

7. DocMaps. Cited [2021 Dec 10]. Available from: https://docmaps.knowledgefutures.org/

8. Yan, V., (2021). ReimagineReview data (Version 5) [Data set]. Zenodo. https://doi.org/10.5281/zenodo.5618257

9. Saderi, D., Greaves, S., (2021) Using preprint reviews to drive journal peer review. ASAPbio. Cited [2021 Dec 10]. Available from:

https://asapbio.org/using-preprint-reviews-to-drive-journal-peer-review

10. The Editors of the Lancet Group. (2020). Learning from a retraction. The Lancet 396:10257 https://doi.org/10.1016/S0140-6736(20)31958-9

11. List, B. (2017). Crowd-based peer review can be good and fast. Nature 546: 9 https://doi.org/10.1038/546009a

12. O'Grady, K. (2021). Fifteen journals to outsource peer-review decisions. Science. https://doi.org/10.1126/science.abj0447 